\title{
Polytropic neutron star - black hole merger simulations with a Paczyński-Wiita potential
}

\author{
M. Ruffert ${ }^{2,1}$ and H.-Th. Janka ${ }^{1}$ \\ 1 Max-Planck-Institut für Astrophysik, Karl-Schwarzschild-Str. 1, 85748 Garching bei München, Germany \\ e-mail: thj@mpa-garching.mpg.de \\ 2 The School of Mathematics and the Maxwell Institute, University of Edinburgh, King's Buildings, Edinburgh EH9 3JZ, UK \\ e-mail: m.ruffert@ed.ac.uk
}

Received 22 June 2009 / Accepted 18 January 2010

\section{ABSTRACT}

\begin{abstract}
Context. Mergers of neutron stars (NS) and black holes (BH) are among the strongest sources of gravitational waves and are potential central engines for short gamma-ray bursts.

Aims. We aim to compare the general relativistic (GR) results of other groups with Newtonian calculations of models with equivalent parameters. We vary the mass ratio of the NS to BH and the compactness of the NS. The mass of the NS is $1.4 M_{\odot}$. We compare the dynamics in the parameter-space regions where the NS is expected to reach the innermost stable circular orbit (ISCO) before being tidally disrupted (mass shedding, MS), and vice versa.

Methods. The hydrodynamics is evolved by a Newtonian PPM scheme with four levels of nested grids. We use a polytropic EoS $(\Gamma=2)$, as adopted in the GR simulations. However, instead of full GR we use a Newtonian potential supplemented by a PaczyńskiWiita-Artemova potential for the $\mathrm{BH}$, both disregarding and including rotation of the $\mathrm{BH}$.

Results. If the NS is compact $(C=0.18)$, it is accreted by the BH more quickly, and only a small amount of mass remains outside the $\mathrm{BH}$. If the mass ratio is small $(Q=2$ or 3 ) or the NS is less compact ( $C=0.16$ or less), the NS is tidally torn apart before being accreted. Although most of the mass is absorbed by the $\mathrm{BH}$, some $0.1 M_{\odot}$ remain in a tidal arm. For small mass ratios $(Q=2$ and 3 ), the tidal arm can wrap around the $\mathrm{BH}$ to form a thick disk. When including the effects of either BH spin-up or spin-down by the accreted matter, more mass remains in the surroundings $\left(0.2-0.3 M_{\odot}\right)$.

Conclusions. Although details and quantitative results differ, the general trends of our Newtonian calculations are similar to the GR calculations. A clear delimiting line separating the ISCO from the MS cases is not found. Inclusion of BH rotation as well as sufficient numerical resolution are extremely important.
\end{abstract}

Key words. hydrodynamics - black hole physics - methods: numerical - binaries: close - stars: neutron - accretion, accretion disks

\section{Introduction}

Merging binaries of neutron stars (NS) and black holes (BH) are of interest both as possible central engines for short gamma-ray bursts and as promising gravitational wave sources detectable by the interferometer observatories. As computational power and numerical schemes have progressed so has the detail of simulations of these mergers. Early simulations by our group (Janka et al. 1999) were developed to investigate gamma-ray burst scenarios. We included detailed microphysics (equation of state, neutrino source terms) but accounted for general relativistic effects either only partially (gravitational wave emission and backreaction) or phenomenologically (Paczyński-Wiita potential for the $\mathrm{BH}$ ). Other groups interested in gravitational wave aspects have published results of NS-BH mergers with general relativistic (GR) physics included to different levels of approximation (Etienne et al. 2009; Shibata et al. 2009; Rantsiou et al. 2008; Taniguchi et al. 2008; Duez et al. 2008), but with some variant of the polytropic equation-of-state (EoS) for the neutron star matter. Rantsiou et al. (2008) did not include dynamic GR in that they used a fixed background. Ferrari et al. (2009) investigated quasi-equilibrium sequences of ellipsoidal NSs also including microphysical EoSs in the tidal approximation. A good source of references are the reviews of Faber (2009) and Duez (2010).
We compare the results of a partially post-Newtonian calculation of NS-BH mergers with published GR simulations, to address in particular the following aspect: Miller (2005) pointed out that during the NS-BH binary orbital inspiral, the innermost stable circular orbit (ISCO) can be reached before the NS becomes tidally disrupted; this was confirmed by Taniguchi et al. (2008) and Ferrari et al. (2009). In this case, it is to be expected that most of the NS matter is swallowed by the $\mathrm{BH}$ before the material can spread out and form a massive torus. Since the ISCO is a purely general relativistic effect, its consequences will not be seen in a purely Newtonian simulation. However, the Paczyński-Wiita potential (Paczyński \& Wiita 1980; Abramowicz 2009) phenomenologically mimics the ISCO in a Newtonian setting. So the question arises as to whether and to what extent one can reproduce the results of the general relativistic simulations with crude Newtonian approximations.

Which of the two cases of NS reaching ISCO first or NS becoming tidally disrupted first happens during the evolution will have a direct impact upon the amount of mass remaining in the torus around the BH. This mass is supposed to provide the energy for the jet of the short-duration gamma-ray bursts, so a lower mass should produce a dimmer burst. Intrinsically, the tidal disruption - called "mass shedding" (MS) in previous papers - should yield a more massive torus than the ISCO case. As shown in our previous investigations (Ruffert et al. 2001 and 
references therein, Setiawan et al. 2006), fairly massive tori of at least several hundredths of a solar mass seem to be needed for the scenario of a hot neutrino-emitting disk to function as a central engine for gamma-ray bursts (Lee et al. 2005; Oechslin \& Janka 2006). Thus the question of how the NS becomes tidally disrupted or swallowed by the $\mathrm{BH}$, and how much mass remains inside the torus is an important one to explain gamma-ray bursts that needs to be investigated in detail. The results of previously published GR simulations still disagree with each other in this point.

Following the introduction above, this paper will describe the methods in the next section and the results of the simulations in Sect. 3. These results are then discussed (Sect. 4) and the paper concludes with a summary (Sect. 5).

\section{Methods}

\subsection{Theory and numerical formulation}

The three-dimensional hydrodynamic simulations were performed with a basically Newtonian code based on the piecewise parabolic method (PPM) of Colella \& Woodward (1984) with four levels of nested grids (Ruffert 1992). The equidistant Cartesian grids all have the same number of zones, depending on the model, either 128 or 256 zones per dimension. Initially the finest grid covers both components of the system (neutron star and black hole). We use an ideal gas equation-of-state (EoS, $P=(\Gamma-1) \epsilon$ with adiabatic index $\Gamma=2$, pressure $P$, and internal energy density $\epsilon$ ) to close the energy equation evolved in the hydrodynamics code. The self-gravity of the gaseous mass distribution on the grids are calculated using fast-Fourier transforms (FFT). Details of the implementation of the nested grids can be found in Ruffert (1992). A low density medium, of roughly $10^{-8}$ the central density of the NS, fills the volume outside the NS and the BH. This "atmosphere" is necessary for numerical reasons (e.g., use of the reciprocal density) and is common to grid-based codes, both for Newtonian and GR simulations. The density of all zones is kept above this minimal value, which is referred to as "vacuum".

The black hole $(\mathrm{BH})$ of mass $M_{\mathrm{BH}}$ is treated as a gravitational point mass surrounded by a vacuum sphere discretised on the grids. The existence of the innermost stable circular orbit (ISCO) is mimicked by including a Paczyński-Wiita-type potential (Paczyński \& Wiita 1980; Artemova et al. 1996). The Artemova et al. prescription for the acceleration is

$\frac{\mathrm{d} \Phi_{\mathrm{BH}}}{\mathrm{d} r}=-\frac{G M_{\mathrm{BH}}}{r^{2-\beta}\left(r-r_{\mathrm{H}}\right)^{\beta}}$,

where $r_{\mathrm{H}}$ is the black hole event horizon, and $\beta$ is a constant for a given value of the $\mathrm{BH}$ spin parameter $a$. It is defined by $\beta(a)=\left(r_{\text {in }}(a)-r_{\mathrm{H}}(a)\right) / r_{\mathrm{H}}(a)$, where $r_{\text {in }}(a)$ is the radius of the ISCO. This prescription reduces to the usual Paczyński-Wiita potential when the $\mathrm{BH}$ spin parameter $a$ is zero. We then have that $\beta=2$, and $r_{\mathrm{H}}(a=0)=2 G M_{\mathrm{BH}} / c^{2}$. We note a mathematical discontinuity: for $\beta>0, r_{\mathrm{H}}$ remains a lower limit for $r>r_{\mathrm{H}}$, whereas for $\beta=0$ one recovers the Newtonian case for all $r>0$ (see Fig. 1). The graphs of $r_{\mathrm{H}}(a), \beta(a)$, and $r_{\text {in }}(a)$ can be found in Setiawan et al. (2006), their Fig. 1.

This potential is implemented numerically as follows. First the total Newtonian potential is calculated for all gas on the grids, including the $\mathrm{BH}$ mass. From this potential, the accelerations are derived by discrete differencing, as is common procedure. We then add the acceleration given by the difference

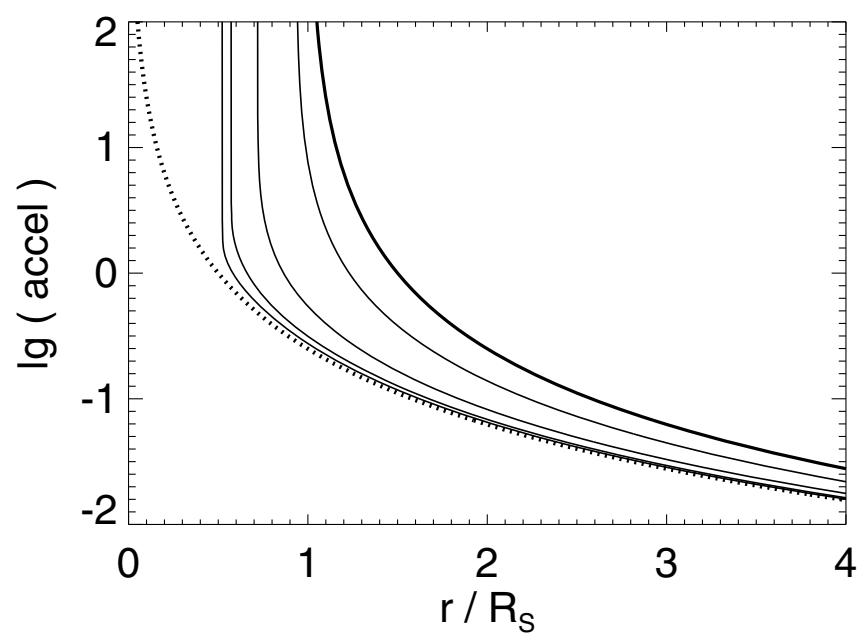

Fig. 1. Gravitational acceleration as a function of distance $r$, as given by the prescription of Eq. (1), for various spin parameters $a=0.999$, 0.99, 0.9, 0.5, 0; solid lines from left to right. The dotted line shows a Newtonian potential acceleration $\propto r^{-2}$. The bold solid line shows the Paczyński-Wiita case $\propto\left(r-R_{\mathrm{S}}\right)^{-2}$, i.e., $a=0 . R_{\mathrm{S}}$ is the Schwarzschild radius.

between the formalism of Eq. (1) and a purely Newtonian potential. By construction, this difference only acts on the gaseous NS matter on the grid. To respect Newton's Third Law ("action = reaction"), a tab of the sum of these accelerations has to be kept and its inverse enforced on the $\mathrm{BH}$.

The radius of the vacuum sphere mimicking the $\mathrm{BH}$ is chosen to be the arithmetic mean of the event horizon and the ISCO. The mass, momentum, and angular momentum of material flowing across this inner boundary are added to the $\mathrm{BH}$ values and the zones affected are reset to near-vacuum values. The position of the $\mathrm{BH}$ is adjusted accordingly - the centre-of-mass of the gas leaving the grid and of the $\mathrm{BH}$ do not necessarily coincide - and moved by a leapfrog procedure (alternate position and velocity updates). In all models listed in Table 1, the angular momentum of the accreted material does not change the spin parameter $a$ in the equations that determine the $\mathrm{BH}$ potential, Eq. (1); i.e., the $\mathrm{BH}$ potential only deepens because of the increase in mass of the $\mathrm{BH}$. We evolved an additional set of four models to investigate the effects of BH spin. These models are listed in Table 2 and discussed in separate paragraphs.

The general relativistic effect that is modelled phenomenologically by the procedures described above is the presence of the ISCO and its change in radius. Frame-dragging and many other effects, e.g., relativistic kinematics, redshift, time dilation, are omitted. However, we do include the local effects of gravitational-wave emission - the volume integral of which reproduces the quadrupole approximation - and the corresponding back-reaction on the hydrodynamic flow (for details see Ruffert et al. 1996).

\subsection{Initial conditions}

The initial distance of NS to BH varies from model to model, to ensure roughly 2-3 orbits before coalescence (see Table 1). For the finest grid to cover both components of the binary, the geometric extent of the grids has to be adapted to ensure the highest possible numerical resolution. Both the extent of the largest grid as well as the size of the finest zone are listed in Table 1.

Parameters for the NS model are given as follows. The mass is kept constant for all polytropic models, $M_{\mathrm{NS}}=1.4 \mathrm{M}_{\odot}$. 
Table 1. Key initial model parameters and some results.

\begin{tabular}{cccccccccccc}
\hline \hline Model & $Q$ & $C$ & $\begin{array}{c}M_{\mathrm{BH}} \\
M_{\odot}\end{array}$ & $\begin{array}{c}R_{\mathrm{NS}} \\
\mathrm{km}\end{array}$ & $\begin{array}{r}d_{\mathrm{i}} \\
\mathrm{km}\end{array}$ & $\begin{array}{r}L \\
\mathrm{~km}\end{array}$ & $\begin{array}{c}\Delta x \\
\mathrm{~km}\end{array}$ & $\begin{array}{c}M_{\mathrm{u}} \\
M_{\odot}\end{array}$ & $\begin{array}{c}M_{\mathrm{b}} \\
M_{\odot}\end{array}$ & $\begin{array}{c}M_{\mathrm{g}} \\
M_{\odot}\end{array}$ & $|\mathrm{h} / \mathrm{l}|$ \\
\hline M5.145 & 5 & 0.145 & 7.0 & 14.3 & 92 & 1550 & 0.77 & 0.07 & 0.08 & 0.15 & $\mathrm{~N}$ \\
M4.145 & 4 & 0.145 & 5.6 & 14.3 & 80 & 1400 & 0.68 & 0.08 & 0.10 & 0.18 & $\mathrm{Y}$ \\
M3.145 & 3 & 0.145 & 4.2 & 14.3 & 68 & 1100 & 0.54 & 0.06 & 0.09 & 0.15 & $\mathrm{Y}$ \\
M2.145 & 2 & 0.145 & 2.8 & 14.3 & 56 & 880 & 0.43 & $<10^{-2}$ & 0.07 & 0.08 & $\mathrm{Y}$ \\
M5.160 & 5 & 0.160 & 7.0 & 12.9 & 92 & 1550 & 0.77 & 0.01 & 0.05 & 0.06 & - \\
M4.160 & 4 & 0.160 & 5.6 & 12.9 & 76 & 1250 & 0.61 & 0.03 & 0.06 & 0.09 & $\mathrm{~N}$ \\
M3.160 & 3 & 0.160 & 4.2 & 12.9 & 66 & 1100 & 0.54 & 0.03 & 0.06 & 0.09 & $\mathrm{~N}$ \\
M2.160 & 2 & 0.160 & 2.8 & 12.9 & 52 & 820 & 0.40 & $<10^{-2}$ & 0.04 & 0.05 & $\mathrm{Y}$ \\
M5.180 & 5 & 0.180 & 7.0 & 11.5 & 88 & 1500 & 0.73 & $<10^{-3}$ & $<10^{-3}$ & $<10^{-3}$ & $\mathrm{~N}$ \\
M4.180 & 4 & 0.180 & 5.6 & 11.5 & 76 & 1250 & 0.61 & $<10^{-3}$ & $<10^{-3}$ & $<10^{-3}$ & $\mathrm{~N}$ \\
M3.180 & 3 & 0.180 & 4.2 & 11.5 & 64 & 1050 & 0.51 & $<10^{-2}$ & 0.01 & 0.01 & $\mathrm{~N}$ \\
M2.180 & 2 & 0.180 & 2.8 & 11.5 & 50 & 780 & 0.38 & $<10^{-3}$ & 0.004 & 0.004 & $\mathrm{~N}$ \\
\hline
\end{tabular}

Notes. Initial parameters: mass ratio $Q$, compactness $C$, black hole mass $M_{\mathrm{BH}}$, neutron star radius $R_{\mathrm{NS}}$, initial orbital distance $d_{\mathrm{i}}$, size of largest grid $L$, size of finest zone $\Delta x=L / 2048$. The mass of the NS is $1.4 M_{\odot}$ in all cases. Values at the end of the simulation, only for high-resolution models: unbound+ejected gas mass $M_{\mathrm{u}}$, bound neutron star mass around BH $M_{\mathrm{b}}$, neutron star mass not instantly accreted by BH $M_{\mathrm{g}},|\mathrm{h} / \mathrm{l}|$ states whether the difference between values for $M_{\mathrm{g}}$ in low-resolution and high-resolution simulations is less than 20\% (Yes/No; '-' no low-res model computed). The spin-up of the BH by accretion of matter is not taken into account.

Table 2. Key initial model parameters and some results for models including BH spin.

\begin{tabular}{cccccccccccccc}
\hline \hline Model & $Q$ & $C$ & $\begin{array}{c}M_{\mathrm{BH}} \\
M_{\odot}\end{array}$ & $a_{\mathrm{i}}$ & $\begin{array}{r}R_{\mathrm{NS}} \\
\mathrm{km}\end{array}$ & $\begin{array}{r}d_{\mathrm{i}} \\
\mathrm{km}\end{array}$ & $N$ & $\begin{array}{r}L \\
\mathrm{~km}\end{array}$ & $\begin{array}{r}\Delta x \\
\mathrm{~km}\end{array}$ & $\begin{array}{c}M_{\mathrm{u}} \\
M_{\odot}\end{array}$ & $\begin{array}{c}M_{\mathrm{b}} \\
M_{\odot}\end{array}$ & $\begin{array}{c}M_{\mathrm{g}} \\
M_{\odot}\end{array}$ & $\begin{array}{c}a_{\mathrm{f}} \\
\mathrm{km}\end{array}$ \\
\hline R5.180 & 5 & 0.180 & 7.0 & 0.00 & 11.5 & 88 & 256 & 1450 & 0.71 & 0.11 & 0.07 & 0.18 & 0.39 \\
$\mathrm{R} 3.160$ & 3 & 0.160 & 4.2 & 0.00 & 14.3 & 66 & 256 & 1100 & 0.54 & 0.08 & 0.14 & 0.22 & 0.44 \\
$\mathrm{R} 2.145$ & 2 & 0.145 & 2.8 & 0.00 & 14.3 & 56 & 128 & 880 & 0.86 & 0.05 & 0.17 & 0.22 & 0.56 \\
$\mathrm{~S} 2.145$ & 2 & 0.145 & 2.8 & 0.99 & 14.3 & 52 & 128 & 850 & 0.83 & 0.02 & 0.26 & 0.28 & 0.88 \\
\hline
\end{tabular}

Notes. Initial parameters: mass ratio $Q$, compactness $C$, black hole mass $M_{\mathrm{BH}}$, initial BH spin parameter $a_{\mathrm{i}}$, neutron star radius $R_{\mathrm{NS}}$, initial orbital distance $d_{\mathrm{i}}$, number of zones per dimension $N$, size of largest grid $L$, size of finest zone $\Delta x=L / 8 N$. The mass of the NS is $1.4 M_{\odot}$ in all cases. Values at the end of the simulation: unbound+ejected gas mass $M_{\mathrm{u}}$, bound neutron star mass around $\mathrm{BH} M_{\mathrm{b}}$, neutron star mass not instantly accreted by BH $M_{\mathrm{g}}$, final BH spin parameter $a_{\mathrm{f}}$.

The radius, $R_{\mathrm{NS}}$, is then fixed by the chosen compactness, $C=$ $\left(G M_{\mathrm{NS}}\right) /\left(c^{2} R_{\mathrm{NS}}\right)$. For a $\Gamma=2$ polytrope, the relation between pressure $P$ and density $\rho$ is $P=\kappa \rho^{2}$, where $\kappa=\frac{2}{\pi} G R_{\mathrm{NS}}^{2}$ is fixed by the NS radius. The density distribution is $\rho(\xi)=\rho_{c} \frac{\sin (\xi)}{\xi}$, with $M_{\mathrm{NS}}=\frac{4}{\pi} R_{\mathrm{NS}}^{3} \rho_{c}$, and $\xi=\pi r / R_{\mathrm{NS}}$.

The main variable of the $\mathrm{BH}$, its mass, is defined in terms of its mass ratio with respect to the NS, $Q=M_{\mathrm{BH}} / M_{\mathrm{NS}}$. Both the NS and BH are initially given Keplerian velocities plus a small radial velocity component from the quadrupole approximation of gravitational wave emission.

In our Newtonian framework, there is no ambiguity about which masses and radii to use in the equations above. This differs from relativistic calculations, where a choice of masses (e.g., $\mathrm{ADM}$, rest), and distances (e.g., isotropic, circumferential) exists. We return to this point when trying to compare our results with other people's simulations, in Sect. 4.

When the NS is evolved in the simulation, the initially spherical NS adapts to the BH potential, orbital motion, and grid resolution on its (NS) dynamical timescale, i.e., within much less than one orbital revolution. This initial phase is almost complete by the time the NS crosses the ISCO or becomes tidally disrupted.

The actual values of the mass ratio $Q$ and compactness $C$ can be found in Table 1. They are also shown in Fig. 2 and were chosen to match closely the models presented in Shibata et al. (2009) (see Fig. 3). Figures 2 and 3 include the line separating the regions within which the NS first reaches the innermost stable circular orbit (ISCO) and the mass shedding limit (MS), respectively. It is taken from Taniguchi et al. (2008) and is based on quasi-equilibrium configurations of the binary system.

\section{Results}

\subsection{Resolution and convergence}

Figure 4 shows results for both high-resolution and lowresolution models. Simulations with lower mass ratios and less compact NSs have intrinsically better resolution (see Table 1). The dynamics for these models and the results, e.g., gas mass on the grid after merger, agree reasonably well for the high- and low-resolution calculations. Thus $128^{3}$ runs, which are computationally feasible in a few days, yield converged results. However, the models with very compact NSs - which have comparatively fewer zones that cover the NS volume and in particular the steep surface gradients - or models with a high mass ratio - i.e., a large BH, which in turn implies large zones - require the use of at least $256^{3}$ zones to be adequately resolved. This can also be seen in Fig. 6, for example: for model M2.145, both the lowand high-resolution simulations can hold the NS intact on the grid and thus the maximum density during the first few orbits are equal. On the other hand, the maximum density of the NS in model M4.180 is distinctly lower in the low-resolution simulation than in the high-resolution calculation, which indicates that the NS develops a more compact core during the initial orbital motion. For very low resolution models, the NS core dissolves 


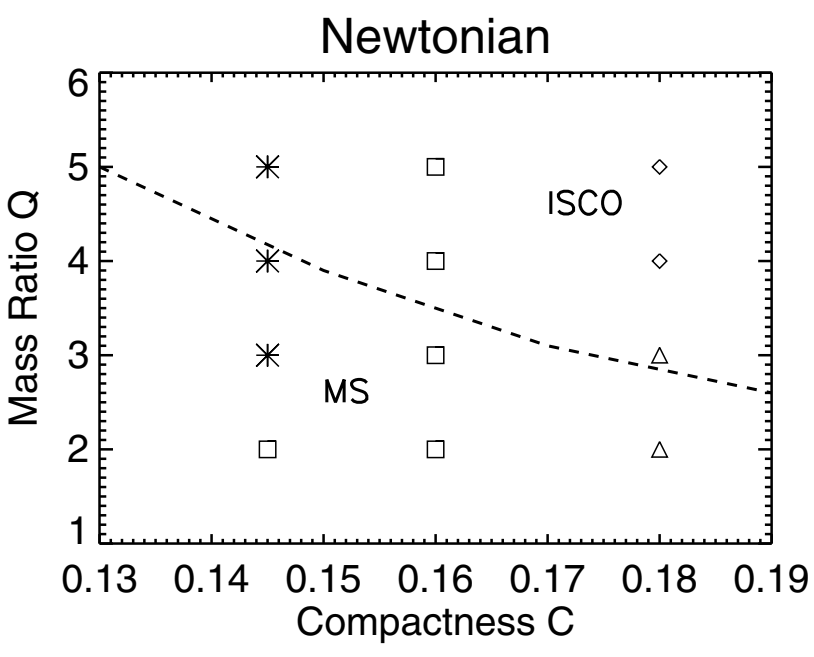

Fig. 2. Parameter space of models presented in this paper (only nonspinning $\mathrm{BH})$, with compactness $C=\left(G M_{\mathrm{NS}}\right) /\left(c^{2} R_{\mathrm{NS}}\right)$ and mass ratio $Q=M_{\mathrm{BH}} / M_{\mathrm{NS}}$. Each symbol represents one model: "diamond" $M_{\mathrm{g}}<$ $10^{-3} M_{\odot}$, "triangle" $10^{-3} M_{\odot}<M_{\mathrm{g}}<0.02 M_{\odot}$, "square" $0.02 M_{\odot}<$ $M_{\mathrm{g}}<0.1 M_{\odot}$, "star" $0.1 M_{\odot}<M_{\mathrm{g}}$, for the mass $M_{\mathrm{g}}$ not instantly accreted by the BH. Note that the spin-up of the BH by the accretion of matter is not taken into account. The line separating the innermost stable circular orbit (ISCO) and the mass shedding (MS) regions is taken from Taniguchi et al. (2008).

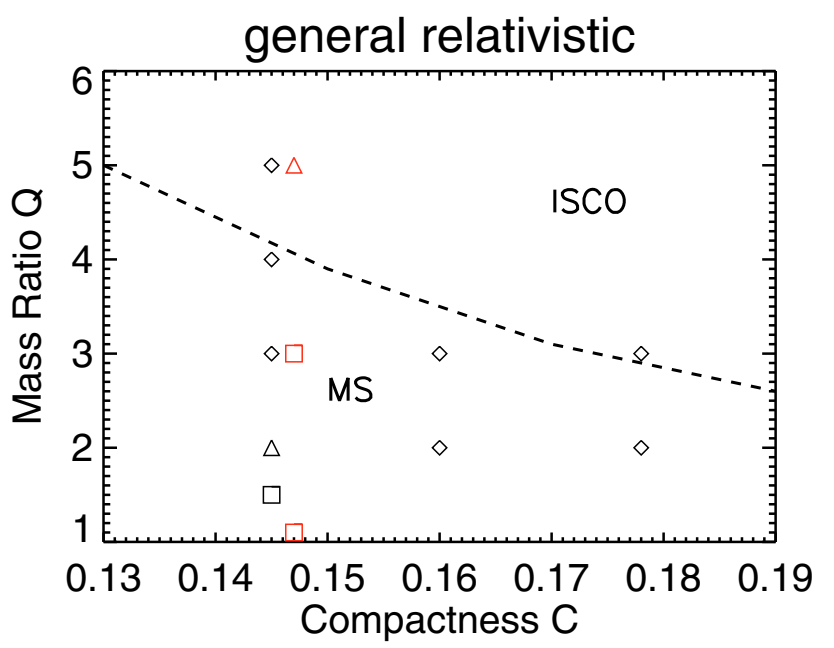

Fig. 3. Results of Shibata et al. (2009) and Etienne et al. (2009) (only non-spinning $\mathrm{BH}$ ) models, with compactness $C$ and mass ratio $Q$. Each symbol represents one model; we use the same symbols as used in Fig. 2. The symbols for the Etienne et al. (2009) models are slightly shifted to $C=0.147$ (and coloured), for clarity.

secularly. For this model the results of the low-resolution simulation should be interpreted with caution. We have no reason to believe that the high-resolution simulations are compromised in this way, but are unable to perform even higher-resolution calculations to check this point.

One notices that even for the simulations where the NS-BH distance is initially identical, the high resolution models reach the point of merger systematically earlier than the low resolution cases. This clearly numerical effect seems to decelerate the radial inward motion of the lower resolution runs, even in the cases resolved best $(Q=2)$ where the central densities for both resolutions match (model M2.145 in Fig. 6). A detailed analysis of the possible cause(s) of this more rapid pre-merging evolution of more highly resolved models did not provide an unambiguous conclusion. We could identify differences in neither the gravitational-wave energy loss, nor the quality of energy conservation, nor the precision of the gravitational potential energy, as clear reason(s) for the observed behaviour. It appears most likely that a difference in angular momentum conservation plays a role. This quantity, however, is very difficult to calculate precisely with its different contributions from gas, orbit, BH spin, and gravitational wave losses, and given the model assumptions (BH spin effects). Therefore, a quantitatively convincing assessment cannot be presented for this possibility.

\subsection{Dynamical evolution}

We describe the merger for the two most extreme cases (nonspinning $\mathrm{BH}$ ) in our set of models:

(1) a small $\mathrm{BH}$ with large $\mathrm{NS}$, specifically a mass ratio of $Q=2$ and a small value for compactness of $C=0.145$;

(2) a large $\mathrm{BH}$ with a small NS, specifically a mass ratio $Q=5$ and a value for compactness of $C=0.180$. These two models cover the main features seen in the simulations, while other models with intermediate values for $Q$ and $C$ are easily recognised as being similar to one or the other of the two cases.

\subsubsection{Low mass ratio, small compactness}

During the initial orbital decay of model M2.145, the central density of the NS remains fairly constant (Figs. 6 and 4). One notices small oscillations originating in the initially spherical NS adapting to the potential of the $\mathrm{BH}$ and the discretisation on the grid. At about $5 \mathrm{~ms}$, the closest distance of the NS density maximum or centre-of-mass is reached (Fig. 5 bottom left; Fig. 7 top left). At this point, matter is transferred to the $\mathrm{BH}$, but at a relatively slow rate compared to the other case. The NS is tidally disrupted, but not before a substantial portion of its matter is given an elliptic orbit taking it temporarily away from the $\mathrm{BH}$ : we note the increase in distance of the density maximum in all cases of low mass ratio and small compactness (models on the lower and left hand side in Fig. 5). We return to this point of orbital widening in Sect. 4.1. The "radius of the BH" shown in Fig. 5 is the arithmetic mean of the event horizon and innermost stable circular orbit, as described in Sect. 2.2. For a non-rotating $\mathrm{BH}$, this is equal to two Schwarzschild radii.

The NS matter spreads out in a tidal spiral arc containing $0.08 M_{\odot}$ (Fig. 7, central left panel $t=9.96 \mathrm{~ms}$ ). Eventually matter falls back along the spiral arc toward the BH. Some of it manages to complete a full circle around the $\mathrm{BH}$ and then collides with the incoming material. At this point, the infalling matter is deflected away from the $\mathrm{BH}$, the circling material spreads out to fill the space and a torus is formed. The point in time when this deflection occurs is marked by a short vertical line in Fig. 4, and one can see that this applies only to models with low mass ratios of $Q=2$ and $Q=3$, for all three values of compactness. In some cases, the high-resolution models were not evolved sufficiently long to see the formation of a disk.

A fraction of the material in the spiral arc is unbound. This amount is also indicated in Fig. 4, by a short piece of nearhorizontal line. The values are listed in Table 1. 
M. Ruffert and H.-Th. Janka: Polytropic neutron star - black hole merger simulations
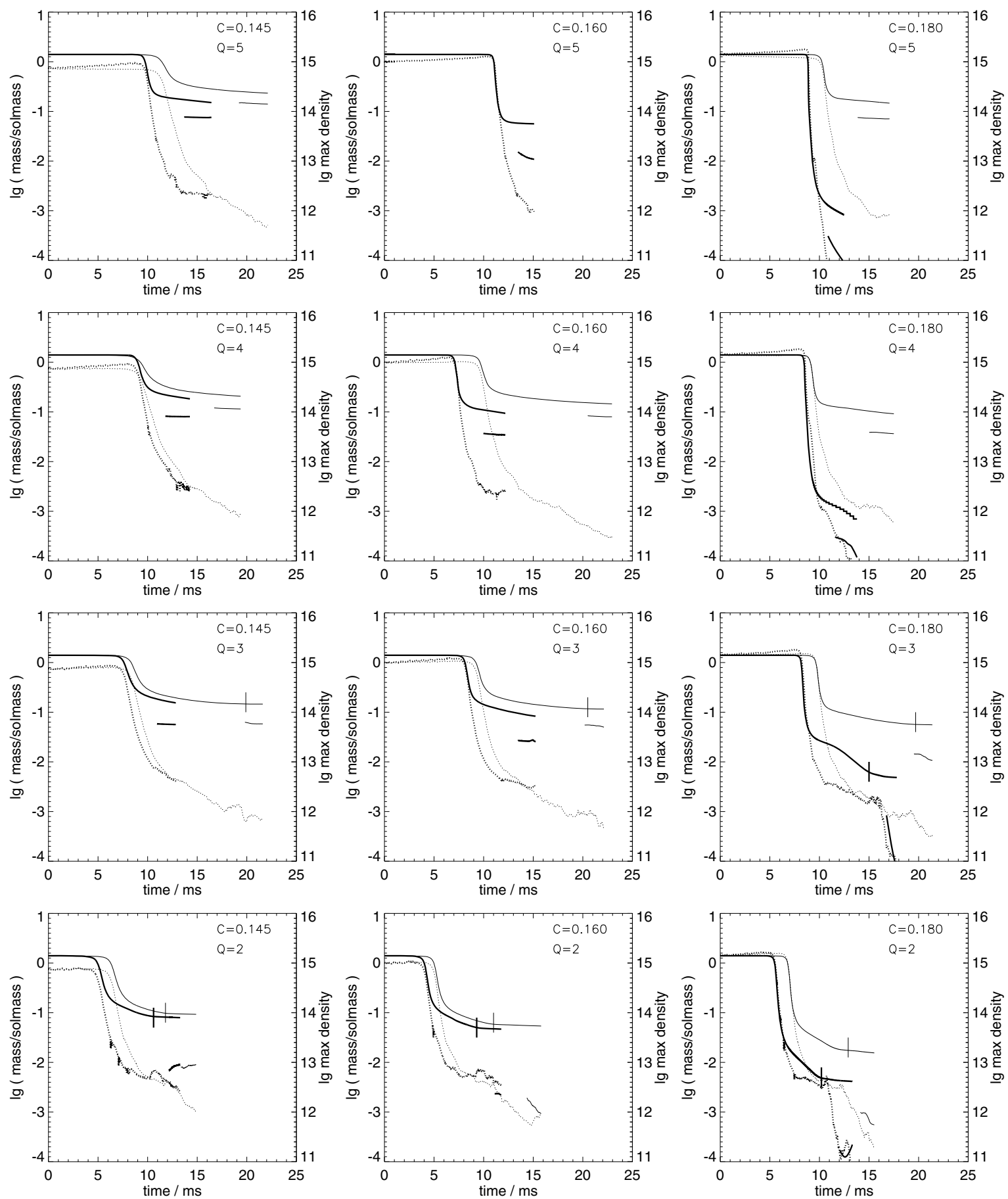

Fig. 4. Temporal evolution of some quantities for various models; the parameter values for $Q$ and $C$ are given within each panel. Solid lines show the gas mass. Dotted lines show the maximum density. Thin lines show low-resolution simulations (128 ${ }^{3}$ ); bold lines show high-resolution simulations $\left(256^{3}\right)$. A short vertical line indicates the formation of an accretion disk. Short pieces of nearly horizontal lines show the amount of unbound mass.

\subsubsection{High mass ratio, large compactness}

During the initial orbital decay of model M5.180, the central density increases substantially (Fig. 6, similarly to model M4.180) in the calculations of sufficiently high resolution. This increase can be noted for all models with massive BHs, i.e., high mass ratios (from close inspection of Fig. 4). So when the NS is close enough to finally transfer mass to the BH, it is very compact and mass transfer proceeds relatively quickly: we note the very steep decline of the mass on the grid for this model
M5.180, in contrast to M2.145 described above. This effect is also partially caused by an increase of the mass and radius providing an essentially direct approach to the absorbing surface for large BHs.

The distance from the $\mathrm{BH}$ at which NS density reaches a maximum decreases continuously all the way to the numerical surface of the BH. In the right panels of Fig. 5, one can see the line of separation that nearly meets the line of $\mathrm{BH}$ radius to within a few $\mathrm{km}$. The minimal distance shown in the opposite case of model M2.145 is much larger, approximately $15 \mathrm{~km}$. 

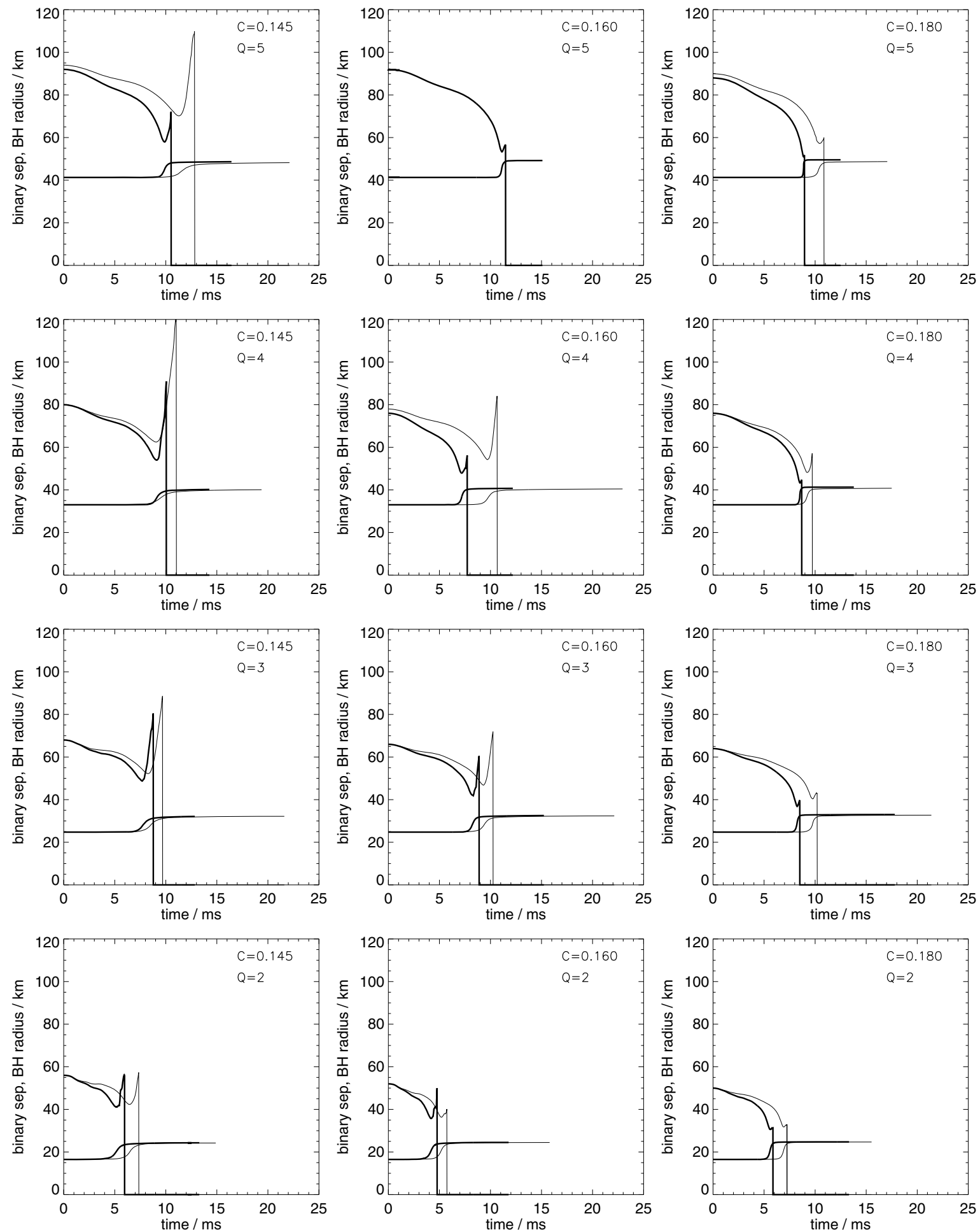

Fig. 5. Temporal evolution of some quantities for various models; the parameter values for $Q$ and $C$ are given within each panel. The upper pair of lines show binary separation; the lower pair of lines show the radius of the $\mathrm{BH}$ (details see text). Thin lines show low-resolution simulations $\left(128^{3}\right)$; bold lines show high-resolution simulations $\left(256^{3}\right)$. The vertical line indicates when the mass within a sphere around the density maximum falls below $0.03 M_{\odot}$.

The NS matter remaining in model M5.180 after the merger, also spreads out in a tidal spiral arc but contains only $10^{-3} M_{\odot}$ (Figs. 4 and 8). The BH, which has a very large extent, accretes all the material that flows back along the arc; none manages to flow all the way around the $\mathrm{BH}$ and form a disk.

Eventually the matter in the outermost portions of the spiral arc reaches the edge of the largest grid (only the lower resolution simulations can be evolved for this length of time). For most models, this happens at around 15-20 ms (depending on the model) at which point the amount of unbound matter ejected from the system is registered by the calculation. The amount of matter ejected is approximately $10^{-3}-10^{-2} M_{\odot}$. We also calculate the amount of material on the grid that is not gravitationally bound, by comparing the internal and kinetic energies with the potential. The values of these mass components are listed in Table 1 and are consistent with the amounts that actually flow off 


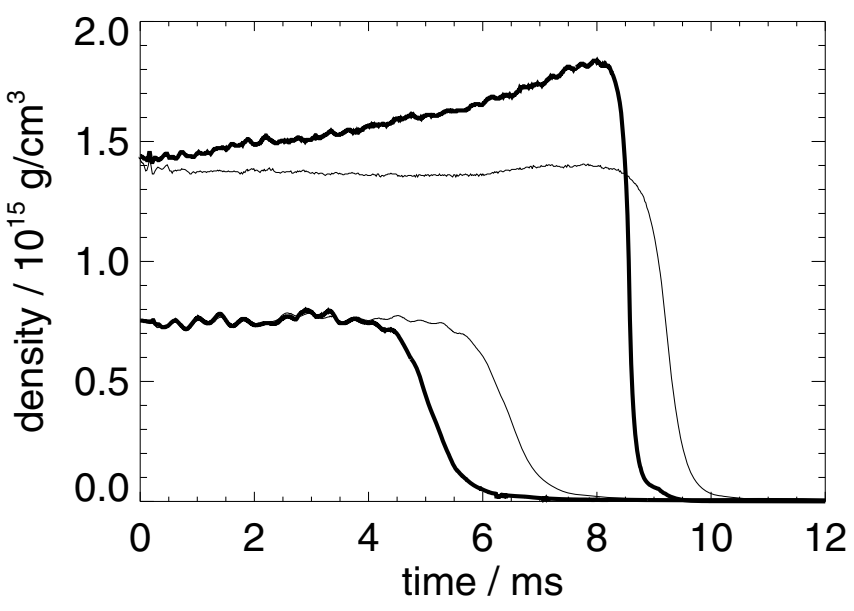

Fig. 6. Maximum density as a function of time for two selected models. The upper pair of lines shows model M4.180; the lower pair of lines shows model M2.145. Thin lines show lower-resolution simulations $\left(128^{3}\right)$; bold lines show higher-resolution simulations $\left(256^{3}\right)$.

the coarsest grid as described above. Dividing the distance travelled by this material - roughly half the grid size $L / 2 \approx 400 \mathrm{~km}-$ by the time elapsed between merger and arriving at the edge of the grid (approximately $5 \mathrm{~ms}$ ), yields an average speed of c/4.

\subsubsection{Comparison of models}

Looking at Figs. 4 and 5, one sees that all models with $C=0.145$ and $C=0.160$ follow the patterns described above for model M2.145. All models with $C=0.180$ follow the mass accretion pattern of M5.180, although some aspects of models M2.180 and M3.180 are different: the BH is small enough in these two cases to allow formation of a disk, albeit with a very small mass. We included the information about the mass remaining on the grids in Fig. 2 in an attempt to outline the similarities between the various models.

\subsection{Effects of $\mathrm{BH}$ spin}

We calculated four models to assess the influence of $\mathrm{BH}$ spin: in three models (R2.145, R3.160, R5.180), the BH is taken to be initially non-rotating $\left(a_{\mathrm{i}}=0\right)$, but we allowed a change of the $\mathrm{BH}$ potential due to spin-up by accretion of angular momentum of the NS matter during the evolution. The opposite case, an initially rapidly spinning BH with $a_{\mathrm{i}}=0.99$, is simulated in model S2.145, and like the other three models, the spin and potential are allowed to change by accretion as well. The complete investigation of the effects of these changes is outwith the remit of this paper and is left to future work. Here we only point out the boundaries of the relevance of results obtained with models ignoring $\mathrm{BH}$ spin.

The post-merger rotational speed of the $\mathrm{BH}$ increases monotonically within the three models with initial $a_{\mathrm{i}}=0 \mathrm{spin}$, from a value of $a_{\mathrm{f}}=0.39$ to $a_{\mathrm{f}}=0.56$ (see Table 2 and Fig. 9) when decreasing the mass ratio and compactness. Following the prescription of Eq. (1), an increasing spin reduces both the radius of the horizon and the radius of the ISCO (Fig. 9). In these models, the decrease in radius caused by spin is greater than the increase caused by mass, or they balance it out, so one observes a slight overall decrease in effective radius (model R5.180) or hardly any change (models R3.160, R2.145): we note the lower near-horizontal line in the right panels of Fig. 9.
If at the closest point when the NS material is being accreted by the $\mathrm{BH}$, the radius of the $\mathrm{BH}$ remains the same or even shrinks - and with it the effective potential - the effectiveness of matter accretion is reduced. In the end, after tidal disruption, much more material remains in the spiral arc than in the equivalent models without BH spin, e.g., compare R5.180 with M5.180. When the models that include the effect of BH spin are completed, between $0.18 M_{\odot}$ and $0.28 M_{\odot}$ of NS material is dispersed around the $\mathrm{BH}$ (see Table 2 and Sect. 4.2 for further discussion).

The initially rapidly rotating $\mathrm{BH}$ (model $\mathrm{S} 2.145$ ) is decelerated from $a_{\mathrm{i}}=0.99$ to $a_{\mathrm{f}}=0.88$ (Table 2 and Fig. 9) because the initial $\mathrm{BH}$ with $a_{\mathrm{i}}=0.99$ has a higher specific angular momentum than the matter that is subsequently accreted. In addition to the increase in mass, the reduction in rotation itself increases the size of the BH: we note the large increase in the circle radius in the right panels in Fig. 8 and the rise of lower solid line in the bottom right panel of Fig. 9.

Model R3.160 is the only one presented in this paper that exhibits a distinctive orbit widening, the lower resolution model even showing repeated mass accretion episodes. In this case, the NS remains self-bound, albeit being less massive, after the first close encounter with the $\mathrm{BH}$. In the high-resolution simulation the NS is tidally disrupted during the second encounter, while for the low-resolution simulation it takes another two episodes before the NS is tidally disrupted. The orbital distance plot (Fig. 9, right panel, second from top) resembles those from our own previous works (Janka et al. 1999) and others (e.g., Davies et al. 2005). We return to this point below.

\section{Discussion}

We discuss three specific points: (1) the orbit widening during the accretion and tidal disruption of the NS; (2) the amount of mass remaining to produce a disk; and (3) the consequences of the NS reaching the ISCO before being tidally disrupted.

\subsection{Orbit widening}

In previous simulations that adopted a purely Newtonian potential for the $\mathrm{BH}$, it was noted that during mass accretion from the NS into the $\mathrm{BH}$, the radial position of the density maximum shifted outwards. This resulted in a lighter NS moving away from the $\mathrm{BH}$ on an elliptic orbit. Of course, the shape of the orbit and the gravitational wave emission eventually caused the NS to experience a second interaction with the $\mathrm{BH}$, in the meantime the accretion, however, being effectively shut off. The sequence of mass transfer and orbit widening can be repeated. Numerically this was observed e.g., in Lee \& Kluźniak (1999, their Fig. 20 shows the binary separation), Janka et al. (1999; their Fig. 1 shows the orbital separation) and in Rosswog et al. (2004; their Fig. 7 shows the mass accretion episodes). It was explained with a semi-analytic description by Davies et al. (2005), in which some "fraction of the angular momentum of the matter (transferred from the NS to the BH) is fed back into the NS". Their Fig. 1 shows the orbital separation.

Miller (2005) argued that the presence of an ISCO, which is a purely relativistic effect, would substantially alter the outcome of a NS-BH merger, since the NS would be preferentially absorbed rather than tidally disrupted. This is basically corroborated by in the general relativistic simulations of Rantsiou et al. (2008), Etienne et al. (2009) and Shibata et al. (2009; their Fig. 1 shows the orbital separation without any sign of orbit widening). 



Fig. 7. The mass density distributions for models M2.145 and M3.180 are displayed in the orbital plane with contours spaced logarithmically in steps of $0.5 \mathrm{dex}$, with units $\mathrm{g} \mathrm{cm}^{-3}$. The arrows indicate the velocity field. The circle at the centre outlines the $\mathrm{BH}$ radius, which is the arithmetic mean of the event horizon and ISCO. 
M. Ruffert and H.-Th. Janka: Polytropic neutron star - black hole merger simulations
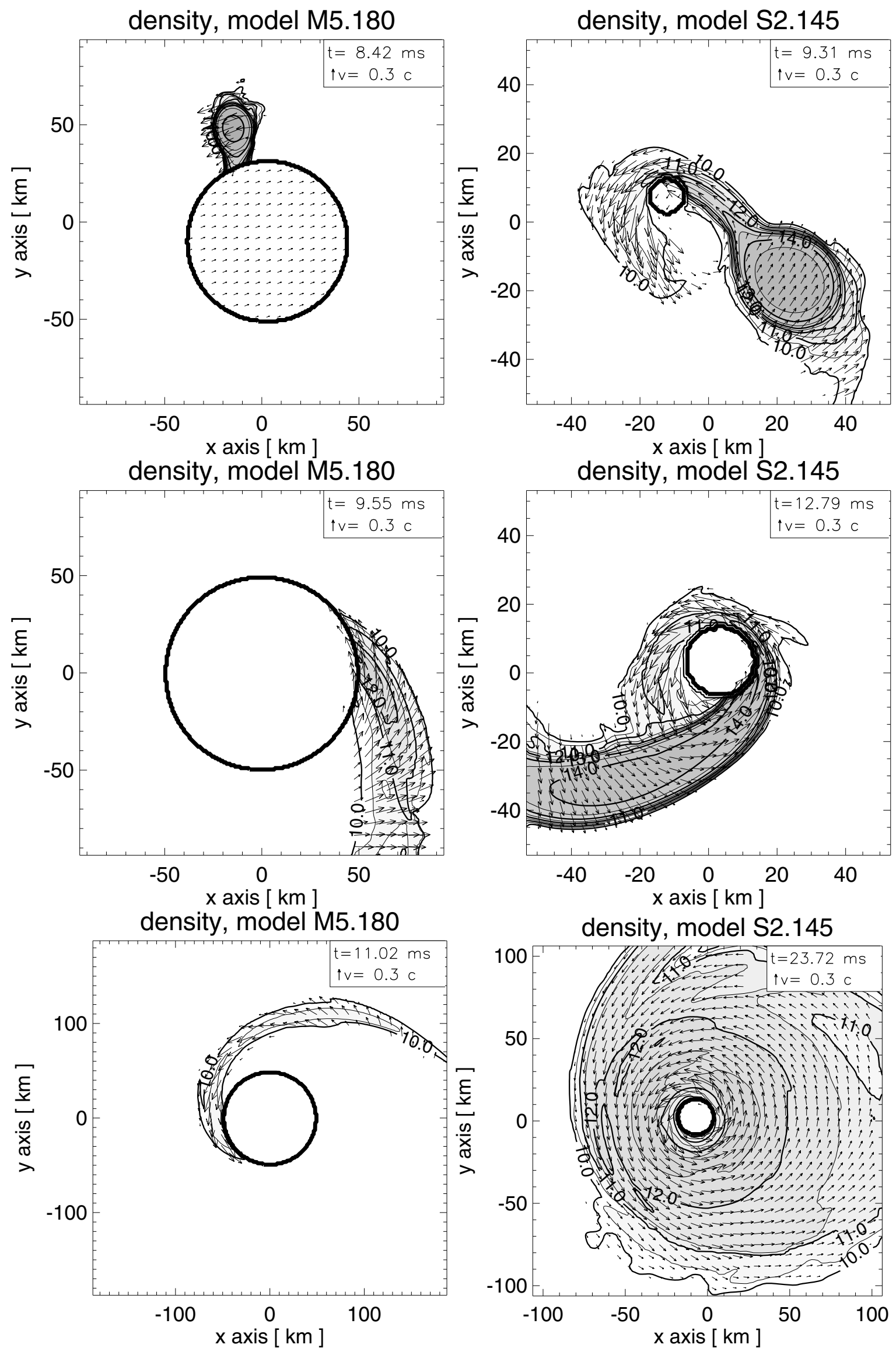

Fig. 8. The mass density distributions for models M5.180 and S2.145 are displayed in the orbital plane with contours spaced logarithmically in steps of $0.5 \mathrm{dex}$, with units $\mathrm{g} \mathrm{cm}^{-3}$. The arrows indicate the velocity field. The circle at the centre outlines the BH radius, which is the arithmetic mean of the event horizon and ISCO. 
A\&A 514, A66 (2010)
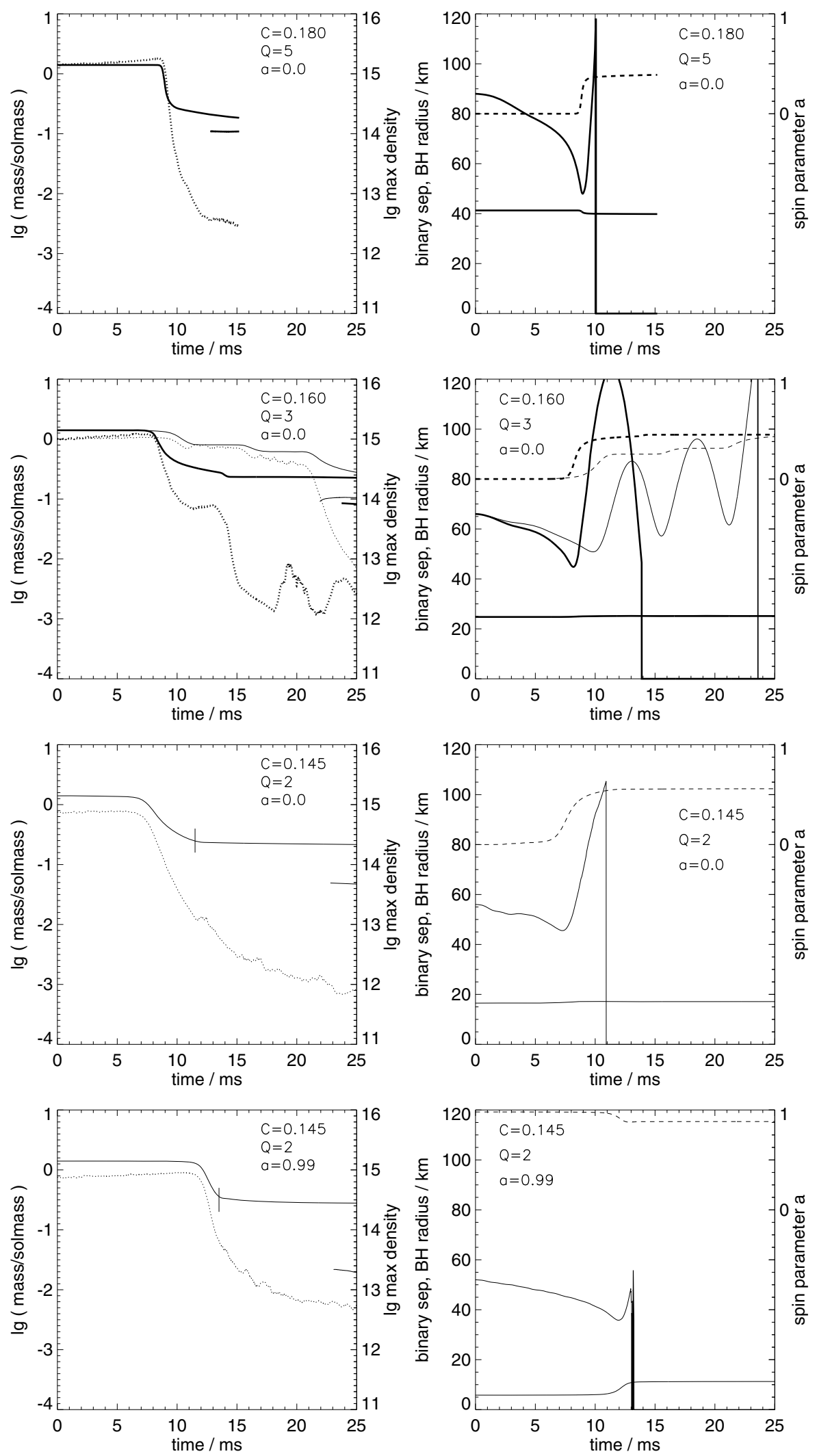

Fig. 9. Temporal evolution for models R5.180, R3.160, R2.145, and S2.145 (from top to bottom), which include the effect of spin-up of the $\mathrm{BH}$ (see Table 2). The initial value of the $\mathrm{BH}$ rotation parameter is given in the panels. Thin lines show lower-resolution simulations $\left(128^{3}\right)$; bold lines show higher-resolution simulations $\left(256^{3}\right)$. The left column of panels shows: the gas mass (solid lines) and the maximum density (dotted lines). A short vertical line indicates the formation of an accretion disk. Short pieces of nearly horizontal lines show the amount of unbound mass. The right column of panels shows: the radius of the $\mathrm{BH}$ (arithmetic mean of horizon and ISCO) as lower solid line, the binary separation as upper solid line, and the BH spin parameter $a$ as top dashed line. The vertical line indicates when the mass within a sphere around the density maximum falls below $0.03 M_{\odot}$.

The Paczyński-Wiita potential in our simulations mimics the ISCO, so the effect outlined by Miller (2005) should be present in our otherwise Newtonian simulations. Indeed, the plots of the distance between the centre of the $\mathrm{BH}$ and the centre-of-mass of the NS (Figs. 5, 9) place the results of our simulations squarely in-between the Newtonian and the relativistic results: we observe a beginning of an orbit widening, but the widening is not fast enough to produce a self-bound "mini"-NS. In particular, in the models with high mass ratios and highly compact NSs, the binary distance shrinks to the "surface" of the BH and hardly any subsequent orbit widening is seen. The NS is practically absorbed whole, as noted in the relativistic calculations. 
An exception is model R3.160 (including the potential changing effects of $\mathrm{BH}$ spin), which exhibits the repeated mass accretion episodes that are very similar to the purely Newtonian models (see Fig. 9 right panel second from top). This might be the consequence of two opposing effects. Firstly, the orbit widening is more pronounced in models that include $\mathrm{BH}$ spin (compare right panels of Fig. 9 with the equivalent models in Fig. 5, because the denser regions of the "escaping" NS are caught up to a lesser degree within the smaller BH radius. Secondly, model R3.160 is intermediate between R5.180 where the large BH swallows up the compact NS, and model R2.145 where a small BH produces a large differential tidal field on an extended NS. In this latter case, the NS is tidally disrupted, although its orbit has widened significantly. The remaining mass in model R3.160 manages to recollect itself into a roughly spherical NS on an elliptic orbit. We finally note, however, that the number of mass-transfer episodes of this model still varies between the simulations of different resolution, so the detailed values and sequence of events (e.g., number of cycles) have to be interpreted with caution.

\subsection{Disk mass}

The amount of material not "instantly" absorbed by the BH but remaining in the surroundings and potentially available to form a torus, is an important energy source for powering the jet of a gamma-ray burst. Thus the questions of how much matter is accreted, how much is ejected, and how much forms a torus, need to be investigated carefully. In this point, the numerical general relativistic simulations (Rantsiou et al. 2008; Etienne et al. 2009; Shibata et al. 2009, their Fig. 7) still differ markedly among themselves, even for models with similar parameters. The range spans from $\approx 0.1 M_{\odot}$ to less than $10^{-4} M_{\odot}$. To power gammaray bursts effectively, at least some hundredths of a solar mass appear to be needed.

The values of mass remaining around the BH for our models are tabulated in Tables 1 and 2. Only in models with both a compact NS $(C=0.180)$ and a non-rotating $\mathrm{BH}$, does most of the matter become accreted quickly by the $\mathrm{BH}$ and only $10^{-2}-10^{-3} M_{\odot}$ remain to form a torus, especially for the massive BHs with a high mass ratio $Q$. This trend matches the general relativistic findings; however, the absolute quantities of mass are higher in our models, e.g., Shibata et al. (2009) obtain typically $1 \%$ of the neutron star mass for models of low mass ratio $Q$. On the other hand, Etienne et al. (2009) infer $0.06 M_{\odot}$ for a nonrotating $\mathrm{BH}$ model with $Q=3$. For less compact NSs, we obtain around $0.1 M_{\odot}$.

As already mentioned above, our models including the effect of BH spin (Table 2) end with a significant amount (between $0.18 M_{\odot}$ and $0.28 M_{\odot}$ ) of NS material spread out around the $\mathrm{BH}$, as the BH's effective potential depth decreases because the spin-up matches or outweighs the mass increase (Fig. 9, models R5.180, R2.145).

\subsection{Tidal disruption vs. ISCO}

Following Miller's (2005) argument, we would expect little mass (tenth of a percent of a solar mass, or less) to remain around the $\mathrm{BH}$ if the NS reaches the ISCO before being tidally shredded. On the other hand, if the NS were tidally disrupted ("mass shedding", MS) before reaching the ISCO, then significant amounts of matter (several percent of a solar mass) would be able to form a torus around the $\mathrm{BH}$. With this in mind, and to be able to compare with previous relativistic simulations, we chose the model parameters plotted in Fig. 2. This graph also uses different symbols to show the varying remaining gas mass around the $\mathrm{BH}$. No clear correlation pattern can be seen between the remaining gas mass and the expected line separating the ISCO from the MS case, except a general trend that the amount of mass is indeed lower toward the right (models with higher compactness). However, we cannot identify a clear step change. This lack of clear demarcation between ISCO and MS is also the case for the models including general relativity (Shibata et al. 2009, Fig. 3).

One point to remember is that the mass ratio $Q$ and compactness $C$ used in this paper, are based on the purely Newtonian, uniquely defined, masses of NS and $\mathrm{BH}$, and radius of the NS. Of course, in the models with general relativistic physics, $Q$ and $C$ have to be defined with a specific choice of the ADM mass, rest mass, circumferential radius, isotropic radius, etc. This complicates the comparison between our Newtonian results shown in Fig. 2 and the general relativistic work of others in Fig. 3. We would expect these differences to produce a shift in the separating line within the plot, but guess it would only be by several tens of percent, and therefore would not qualitatively change the statements.

\subsection{Rotating $\mathrm{BH}$}

For models R5.180, R3.160, R2.145, and S2.145, we note only that a large difference in dynamics and remaining torus mass is present compared to the equivalent non-rotating polytropic models (for details see Sect. 3.3 and the end of Sects. 4.1 and 4.2). It is beyond the scope of this paper to map out the differences in detail over the full parameter space, but this will be the topic of a subsequent investigation. We note that Etienne et al. (2009) report a fairly high value for the torus mass $\left(0.2 M_{\odot}\right)$ in their $Q=3$ model with a spinning BH $(a=0.75)$.

Our values for the final spin parameter of the $\mathrm{BH}(0.39,0.43$, 0.56) match the values obtained by Shibata et al. (2009; their Table III) fairly well, although their values are systematically larger $(0.42,0.56,0.68$, respectively).

In our pseudo-Newtonian simulations, the mass that assembles into a torus or is ejected, shows a very strong sensitivity to the inclusion of the angular momentum gain by the $\mathrm{BH}$ because of the accretion of matter in the merger. This effect, however, should be generically included in the relativistic models of Shibata et al. (2009) and Etienne et al. (2009).

Since the existence of an ISCO is accounted for by the $\mathrm{BH}$ potentials used in our simulations, the tendency towards reaching higher torus and ejecta masses in our models than relativistic simulations (with similar system parameters) requires a different explanation. We hypothesise that the difference could be a consequence of the stronger self-gravity of relativistically described neutron stars compared to the Newtonian objects considered in our simulations. We note how so much more closely the tidal disruption limits of the pseudo-TOV models of Ferrari et al. (2009) shown in their Fig. 1, match the full GR results than the purely Newtonian models. The deeper relativistic potential might impede the disruption of the neutron star and the formation of a tidal arc, so that a greater fraction of the star is directly swallowed by the $\mathrm{BH}$.

\section{Conclusions}

We reach the following conclusions.

1. We do not note any fundamental and discontinuous difference in the dynamics of the models in the ISCO regime as opposed to the "mass shedding" (= tidal disruption) regime. 
The variation in masses that are not instantly accreted by the BH is continuous (Figs. 2 and 3 ).

2. In all cases the NS is initially tidally stretched into an arc, albeit of very different mass in each of the various cases.

3. For models without $\mathrm{BH}$ spin, the onset of orbital widening is noted during the phase of tidal shredding of the NS but is only short-lived.

4. In one case of a model including the BH spin, we see the formation of a "mini" NS on an extended elliptic orbit, followed by episodic mass transfer, and a final tidal disruption.

5. In mergers with compact neutron stars $(C=0.180)$, a very small amount, less than $10^{-2} M_{\odot}$, remains in the surroundings of the $\mathrm{BH}$.

6. Only in mergers of low mass BHs with mass ratio $Q=2$ or $Q=3$, does a closed accretion torus - as opposed to an open arc - form.

7. Mergers with neutron stars of compactness $C=0.145$ or $C=0.160$ produce a significant amount of material (order of $\left.0.1 M_{\odot}\right)$ in the surroundings of the $\mathrm{BH}$.

8. Effects due to $\mathrm{BH}$ spin-up are important and significantly change the results. We note an increase in mass of the material surrounding the $\mathrm{BH}$ of up to $0.2-0.3 M_{\odot}$.

Acknowledgements. We would like to thank the anonymous referee for carefully reading the manuscript and providing helpful and detailed suggestions for improvement of this work.

M.R. would like to thank the Max-Planck-Institut für Astrophysik for the kind hospitality during a sabbatical visit, where this work was accomplished. We thank A. Bauswein for stimulating and helpful discussions. The calculations were performed at the Rechenzentrum Garching (RZG). The project was also supported by the Deutsche Forschungsgemeinschaft through the Transregional
Collaborative Research Centers SFB/TR 27 "Neutrinos and Beyond" and SFB/TR 7 "Gravitational Wave Astronomy", and the Cluster of Excellence EXC 153 (http: //www . universe-cluster . de) "Origin and Structure of the Universe".

\section{References}

Abramowicz, M. A. 2009, A\&A, 500, 213

Artemova, I. V., Björnsson, G., \& Novikov, I. D. 1996, ApJ, 461, 565

Colella, P., \& Woodward, P. R. 1984, J. Comput. Phys., 54, 174

Davies, M. B., Levan, A. J., \& King, A. R. 2005, MNRAS, 356, 54

Duez, M., Foucart, F., Kidder, L., et al. 2008, Phys. Rev. D, 78, 104015

Duez, M. 2010, Class. Quantum Grav., 27, 114002

Etienne, Z. B., Faber, J. A., Liu, Y. T., et al. 2008, Phys. Rev. D, 77, 084002

Etienne, Z. B., Liu, Y. T., Shapiro, S. L., et al. 2009, Phys. Rev. D, 79, 044024

Faber, J. 2009, Class. Quantum Grav., 26, 114004

Ferrari, V., Gualtieri, L., \& Pannarale, F. 2009, Class. Quant. Grav., 26, 125004 Janka, H.-Th., Eberl, T., Ruffert, M., et al. 1999, ApJ, 527, L39

Lee, W. H., \& Kluźniak, W. 1999, ApJ, 526, 178

Lee, W. H., Ramirez-Ruiz, E., \& Granot, J. 2005, ApJ, 630, L165

Miller, M. C. 2005, ApJ, 626, L41

Oechslin, R., \& Janka, H.-Th. 2006, 368, 1489

Paczyński, B., \& Wiita, P. 1980, A\&A, 88, 32

Portegies Zwart, S. F. 1998, ApJ, 503, L53

Rantsiou, E., Kobayashi, S., Laguna, P., et al. 2008, A\&A, 680, 1326

Rasio, F. A., \& Shapiro, S. L. 1999, Class. Quant. Grav., 16, R1

Rosswog, S., Speith, R., \& Wynn, G. A. 2004, MNRAS, 351, 1121

Ruffert, M. 1992, A\&A, 265, 82

Ruffert, M., \& Janka, H.-Th. 2001, A\&A, 380, 544

Ruffert, M., Janka, H.-Th., \& Schaefer, G. 1996, A\&A, 311, 532

Ruffert, M., Janka, H.-Th., Takahashi, K., et al. 1997, A\&A, 319, 122

Setiawan, S., Ruffert, M., \& Janka, H.-Th. 2006, A\&A, 458, 553

Shen, H., Toki, H., Oyamatsu, K., et al. 1998, Nucl. Phys. A, 637, 435

Shibata, M., Kyutoku, K., Yamamoto, T., et al. 2009, Phys. Rev. D, 79, 044030

Taniguchi, K., Baumgarte, T. W., Faber, J. A. et al. 2008, Phys. Rev. D, 77, 044033 